Vol. XX (XXXX)

No. X

\title{
VARIATIONAL INTEGRATORS AND TIME-DEPENDENT LAGRANGIAN SYSTEMS
}

\author{
M. DE LEÓN, D. MARTÍN DE DIEGO* \\ Instituto de Matemáticas y Física Fundamental, CSIC, \\ Serrano 123, 28006 Madrid, Spain \\ (e-mail: mdeleon@imaff.cfmac.csic.es, d.martin@imaff.cfmac.csic.es)
}

(Received 2001)

\begin{abstract}
This paper presents a method to construct variational integrators for timedependent lagrangian systems. The resulting algorithms are symplectic, preserve the momentum map associated with a Lie group of symmetries and also describe the energy variation.
\end{abstract}

Key words: variational integration algorithm, time-dependent lagrangian, symplectic integrator.

\section{Introduction}

There are several numerical integration methods [16] that preserve some of the invariants of an autonomous mechanical system. In [8], T.D. Lee studies the possibility that time can be regarded as a bona fide dynamical variable giving a discrete time formulation of mechanics (see also [9, 10]). From other point of view (integrability aspects) Veselov [19] uses a discretization of the equations of classical mechanics. Both approaches can be characterized as the creation of integrators based on a discretization of the variational principle determined by a lagrangian function. These integration methods have usually better long term simulation properties and computational efficiency than the conventional ones.

The main geometrical invariants that these integrators preserve are symplecticity, energy or/and momentum. Ge and Marsden [4] have proved that a constant time stepping integrator cannot preserve the symplectic form, energy and momentum, simultaneously, unless it coincides with the exact solution of the initial system up to a time reparametrization. However, Kane, Marsden, Ortiz and West [6] show that using an appropriate definition of symplecticity and an adaptative time stepping it is possible to construct a variational integrator which is simultaneously symplectic, momentum and energy preserving.

${ }^{*}$ Research in part supported by grants DGICYT (Spain), PB97-1257 and PGC2000-2191-E. 
The purpose of this paper is to extend the results previously obtained for conservative mechanical systems to the case of time-dependent lagrangian systems following Lee's approach. The method is also based on a discrete variational principle. In a further work, we will test our algorithm in relevant examples and analyze the deep relationship between the continuous and discrete cases.

From the point of view of applications to problems in mechanics (time-dependent harmonic oscillator, Meshchersky's equations in rocketry, for instance), and in control theory, the time-dependent case definitely deserves special attention. Moreover, time-dependent lagrangian systems appear as an indispensable tool in many economic problems 115, 17. In fact, a typical optimization problem in modern economics deals with extremizing the

functional $\int_{0}^{T} D(t) U[f(t, k, \dot{k})] d t$ subject or not to constraints. Here, $D(t)$ is a discount rate factor, $U$ an utility function, $f$ a consumption function and $k$ the capital-labor ratio. Moreover, the time-dependent case is also important because its relation with Classical Field Theory, and in a future work, we will investigate the extension of these ideas in the framework of multisymplectic geometry (see the preliminary work by Marsden, Patrick and Shkoller [14], and [1, 11] for variational systems subjected to constraints and nonholonomic mechanical systems).

\section{Time-dependent Lagrangian systems}

Time-dependent lagrangian theory is sometime studied using an homogeneous formalism. If $L: \mathbb{R} \times T Q \rightarrow \mathbb{R}$ is a time-dependent lagrangian then the corresponding homogeneous lagrangian $\bar{L}: T_{0}(\mathbb{R} \times Q) \rightarrow \mathbb{R}$ is defined by:

$$
\bar{L}(t, q, \dot{t}, \dot{q})=\dot{t} L(t, q, \dot{q} / \dot{t})
$$

(we delete the submanifold $\dot{t}=0$ from the whole tangent space $T(\mathbb{R} \times Q)$ ). ) A solution $(t(s), q(s))$ of the Euler-Lagrange equation for $\bar{L}$ verifies:

$$
\begin{aligned}
& \frac{d}{d s}\left(\frac{\partial L}{\partial \dot{q}}\left(t(s), q(s), \frac{\dot{q}(s)}{\dot{t}(s)}\right)\right)-\dot{t}(s) \frac{\partial L}{\partial q}\left(t(s), q(s), \frac{\dot{q}(s)}{\dot{t}(s)}\right)=0 \\
& \frac{d}{d s}\left(L\left(t(s), q(s), \frac{\dot{q}(s)}{\dot{t}(s)}\right)-\frac{\dot{q}(s)}{\dot{t}(s)} \frac{\partial L}{\partial \dot{q}}\left(t(s), q(s), \frac{\dot{q}(s)}{\dot{t}(s)}\right)\right)-\dot{t}(s) \frac{\partial L}{\partial t}\left(t(s), q(s), \frac{\dot{q}(s)}{\dot{t}(s)}\right)=0
\end{aligned}
$$

Along the submanifold $\dot{t}=1$ these equations represent the Euler-Lagrange equations for $L$ and the non-preservation of the energy function $E_{L}=\dot{q} \partial L / \partial \dot{q}-L$, respectively (see [3], for instance).

\section{The time-dependent variational integrator}

A discrete time dependent lagrangian is a map $L_{d}: \mathbb{R} \times Q \times \mathbb{R} \times Q \rightarrow \mathbb{R}$. Define the action sum $\mathcal{S}:(\mathbb{R} \times Q)^{N+1} \rightarrow \mathbb{R}$ corresponding to the lagrangian $L_{d}$ by

$$
\mathcal{S}=\sum_{k=0}^{N-1}\left(t_{k+1}-t_{k}\right) L_{d}\left(t_{k}, q_{k}, t_{k+1}, q_{k+1}\right),
$$


where $q_{k} \in Q$ and $t_{k} \in \mathbb{R}$ for $0 \leq k \leq N$.

We know that for any product manifold $M_{1} \times \ldots \times M_{a}, T_{\left(x_{1}, \ldots, x_{a}\right)}^{*}\left(M_{1} \times \ldots \times M_{a}\right) \simeq$ $T_{x_{1}}^{*} M_{1} \oplus \ldots \oplus T_{x_{a}}^{*} M_{a}$, for all $x_{i} \in M_{i}, 1 \leq i \leq a$. Therefore, any covector $\alpha \in$ $T_{\left(x_{1}, \ldots, x_{a}\right)}^{*}\left(M_{1} \times \ldots \times M_{a}\right)$ admits a decomposition $\alpha=\alpha_{1}+\ldots+\alpha_{a}$ where $\alpha_{i} \in T_{x_{i}}^{*} M_{i}$, $1 \leq i \leq a$. Thus,

$$
\begin{aligned}
d L_{d}\left(t_{0}, q_{0}, t_{1}, q_{1}\right)= & D_{1} L_{d}\left(t_{0}, q_{0}, t_{1}, q_{1}\right)+D_{2} L_{d}\left(t_{0}, q_{0}, t_{1}, q_{1}\right) \\
& +D_{3} L_{d}\left(t_{0}, q_{0}, t_{1}, q_{1}\right)+D_{4} L_{d}\left(t_{0}, q_{0}, t_{1}, q_{1}\right)
\end{aligned}
$$

since $T_{\left(t_{0}, q_{0}, t_{1}, q_{1}\right)}^{*}(\mathbb{R} \times Q \times \mathbb{R} \times Q)=T_{t_{0}}^{*} \mathbb{R} \oplus T_{q_{0}}^{*} Q \oplus T_{t_{1}}^{*} \mathbb{R} \oplus T_{q_{1}}^{*} Q$ and, in addition, we have

$$
d L_{d}\left(t_{0}, q_{0}, t_{1}, q_{1}\right)=\bar{D}_{1} L_{d}\left(t_{0}, q_{0}, t_{1}, q_{1}\right)+\bar{D}_{2} L_{d}\left(t_{0}, q_{0}, t_{1}, q_{1}\right)
$$

since $T_{\left(t_{0}, q_{0}, t_{1}, q_{1}\right)}^{*}(\mathbb{R} \times Q \times \mathbb{R} \times Q)=T_{\left(t_{0}, q_{0}\right)}^{*}(\mathbb{R} \times Q) \oplus T_{\left(t_{1}, q_{1}\right)}^{*}(\mathbb{R} \times Q)$. From now on, we will use both decompositions $(D$ and $\bar{D})$ of 1 -forms on $(\mathbb{R} \times Q)^{2}$.

The discrete variational principle assures that the solutions of the discrete system must extremize the action sum given fixed points $\left(t_{0}, q_{0}\right)$ and $\left(t_{N}, q_{N}\right)$. Extremizing $\mathcal{S}$ over $t_{k}, q_{k}, 1 \leq k \leq N-1$ leads to the following system of equations:

$$
\begin{gathered}
\left(t_{k+1}-t_{k}\right) D_{2} L_{d}\left(t_{k}, q_{k}, t_{k+1}, q_{k+1}\right)+\left(t_{k}-t_{k-1}\right) D_{4} L_{d}\left(t_{k-1}, q_{k-1}, t_{k}, q_{k}\right)=0 \\
\left(t_{k+1}-t_{k}\right) D_{1} L_{d}\left(t_{k}, q_{k}, t_{k+1}, q_{k+1}\right)-L_{d}\left(t_{k}, q_{k}, t_{k+1}, q_{k+1}\right) d t_{k}+ \\
\quad+\left(t_{k}-t_{k-1}\right) D_{3} L_{d}\left(t_{k-1}, q_{k-1}, t_{k}, q_{k}\right)+L_{d}\left(t_{k-1}, q_{k-1}, t_{k}, q_{k}\right) d t_{k}=0
\end{gathered}
$$

or, equivalently,

$$
\bar{D}_{1}\left[\left(t_{k+1}-t_{k}\right) L_{d}\left(t_{k}, q_{k}, t_{k+1}, q_{k+1}\right)\right]+\bar{D}_{2}\left[\left(t_{k}-t_{k-1}\right) L_{d}\left(t_{k-1}, q_{k-1}, t_{k}, q_{k}\right)\right]=0 .
$$

Take $\bar{L}_{d}\left(t_{k}, q_{k}, h_{k}, q_{k+1}\right)=L_{d}\left(t_{k}, q_{k}, t_{k}+h_{k}, q_{k+1}\right)$, where $h_{k}=t_{k+1}-t_{k}$ is the time step, then

$$
\begin{aligned}
\frac{\partial L_{d}}{\partial t_{k}} & =\frac{\partial \bar{L}_{d}}{\partial t_{k}}-\frac{\partial \bar{L}_{d}}{\partial h_{k}}, \\
\frac{\partial L_{d}}{\partial t_{k+1}} & =\frac{\partial \bar{L}_{d}}{\partial h_{k}} .
\end{aligned}
$$

Therefore the second set of equations is equivalent to:

$$
\begin{aligned}
& h_{k} \frac{\partial \bar{L}_{d}}{\partial t_{k}}\left(t_{k}, q_{k}, h_{k}, q_{k+1}\right)-h_{k} \frac{\partial \bar{L}_{d}}{\partial h_{k}}\left(t_{k}, q_{k}, h_{k}, q_{k+1}\right)-\bar{L}_{d}\left(t_{k}, q_{k}, h_{k}, q_{k+1}\right)+ \\
& +h_{k-1} \frac{\partial \bar{L}_{d}}{\partial h_{k-1}}\left(t_{k-1}, q_{k-1}, h_{k-1}, q_{k}\right)+\bar{L}_{d}\left(t_{k-1}, q_{k-1}, h_{k-1}, q_{k}\right)=0 .
\end{aligned}
$$


Next, we define the discrete energy function as (see Kane et al [6]):

$$
E_{d}(t, x, h, y)=-\frac{\partial}{\partial h}\left[h \bar{L}_{d}(t, x, h, y)\right]=-\bar{L}_{d}(t, x, h, y)-h D_{3} \bar{L}_{d}(t, x, h, y) .
$$

One can also motivate this definition using the discrete variational principle (see subsection 3.2.).

We deduce that equation (1) is equivalent to:

$$
\frac{1}{h_{k}}\left[E_{d}\left(t_{k}, q_{k}, h_{k}, q_{k+1}\right)-E_{d}\left(t_{k-1}, q_{k-1}, h_{k-1}, q_{k}\right)\right]=-\frac{\partial \bar{L}_{d}}{\partial t_{k}}\left(t_{k}, q_{k}, h_{k}, q_{k+1}\right)
$$

which is a "discretization" of the well-known equation $d E_{L} / d t=-\partial L / \partial t$ for continuous time-dependent lagrangian systems, that measures the non-conservation of the energy.

Consider the mapping

$$
\Phi:(\mathbb{R} \times Q)^{2} \longrightarrow(\mathbb{R} \times Q)^{2}
$$

defined by

$$
\Phi\left(t_{k-1}, q_{k-1}, t_{k}, q_{k}\right)=\left(t_{k}, q_{k}, t_{k+1}, q_{k+1}\right), 1 \leq k \leq N-1,
$$

where $q_{k+1}, t_{k+1}$ are implicitly defined by Equations (1). $\Phi$ will be called the discrete flow of the discrete lagrangian $L_{d}$. If the matrix $\left(\bar{D}_{12} L_{d}\right)$ is regular, then the discrete map $\Phi$ exists and is uniquely defined .

\section{Example 1. A single particle in one-dimensional space}

Take the continuous lagrangian $L(t, x, v)=\frac{m}{2} v^{2}-V(t, x)$ and a typical discretization

$$
L_{d}\left(q_{0}, t_{0}, q_{1}, t_{1}\right)=\frac{m}{2}\left(\frac{q_{1}-q_{0}}{t_{1}-t_{0}}\right)^{2}-V\left(\frac{q_{1}+q_{0}}{2}, \frac{t_{1}+t_{0}}{2}\right)
$$

The implicit discrete algorithm is given by:

$$
\begin{aligned}
& m\left(\frac{q_{k+1}-q_{k}}{t_{k+1}-t_{k}}-\frac{q_{k}-q_{k-1}}{t_{k}-t_{k-1}}\right) \\
& =-\frac{1}{2}\left[\left(t_{k+1}-t_{k}\right) \frac{\partial V}{\partial x}\left(\frac{q_{k+1}+q_{k}}{2}, \frac{t_{k+1}+t_{k}}{2}\right)+\left(t_{k}-t_{k-1}\right) \frac{\partial V}{\partial x}\left(\frac{q_{k}+q_{k-1}}{2}, \frac{t_{k}+t_{k-1}}{2}\right)\right], \\
& E_{L}\left(\frac{t_{k+1}+t_{k}}{2}, \frac{q_{k+1}+q_{k}}{2}, \frac{q_{k+1}-q_{k}}{t_{k+1}-t_{k}}\right)-E_{L}\left(\frac{t_{k}+t_{k-1}}{2}, \frac{q_{k}+q_{k-1}}{2}, \frac{q_{k}-q_{k-1}}{t_{k}-t_{k-1}}\right) \\
& =\frac{1}{2}\left[\left(t_{k+1}-t_{k}\right) \frac{\partial V}{\partial t}\left(\frac{q_{k+1}+q_{k}}{2}, \frac{t_{k+1}+t_{k}}{2}\right)+\left(t_{k}-t_{k-1}\right) \frac{\partial V}{\partial t}\left(\frac{q_{k}+q_{k-1}}{2}, \frac{t_{k}+t_{k-1}}{2}\right)\right]
\end{aligned}
$$

where $E_{L}(t, x, v)=\frac{m}{2} v^{2}+V(t, x)$ is the energy function. 


\subsection{Convergence and error analysis for the discrete algorithm}

For simplicity, we suppose that $Q$ is a vector space and choose the following discretization of the lagrangian $L: \mathbb{R} \times T Q \rightarrow \mathbb{R}:$

$$
L_{d}\left(t_{k}, q_{k}, t_{k+1}, q_{k+1}\right)=L\left(\frac{t_{k}+t_{k+1}}{2}, \frac{q_{k}+q_{k+1}}{2}, \frac{q_{k+1}-q_{k}}{t_{k+1}-t_{k}}\right)
$$

In terms of the discrete velocity $v_{k}=\left(q_{k+1}-q_{k}\right) /\left(t_{k+1}-t_{k}\right)$ and the time step $h_{k}$,

$$
\tilde{L}_{d}\left(t_{k}, q_{k}, h_{k}, v_{k}\right)=L\left(t_{k}+\frac{h_{k}}{2}, q_{k}+v_{k} \frac{h_{k}}{2}, v_{k}\right)
$$

Then, equations (11) and (2) become

$$
\begin{aligned}
& \frac{1}{2 h_{k}}\left[h_{k} \frac{\partial \tilde{L}_{d}}{\partial q_{k}}\left(V_{k}\right)+h_{k-1} \frac{\partial \tilde{L}_{d}}{\partial q_{k-1}}\left(V_{k-1}\right)\right]-\frac{1}{h_{k}}\left[\frac{\partial \tilde{L}_{d}}{\partial v_{k}}\left(V_{k}\right)-\frac{\partial \tilde{L}_{d}}{\partial v_{k-1}}\left(V_{k-1}\right)\right]=0 \\
& \frac{1}{h_{k}}\left[v_{k} \frac{\partial \tilde{L}_{d}}{\partial v_{k}}\left(V_{k}\right)-\tilde{L}_{d}\left(V_{k}\right)-v_{k-1} \frac{\partial \tilde{L}_{d}}{\partial v_{k-1}}\left(V_{k-1}\right)-\tilde{L}_{d}\left(V_{k-1}\right)\right] \\
& =-\frac{1}{2 h_{k}}\left[h_{k} \frac{\partial \tilde{L}_{d}}{\partial t_{k}}\left(V_{k}\right)+h_{k-1} \frac{\partial \tilde{L}_{d}}{\partial t_{k-1}}\left(V_{k-1}\right)\right]
\end{aligned}
$$

where $V_{i}=\left(t_{i}, q_{i}, h_{i}, v_{i}\right)$. It is clear that if $h_{k-1} \rightarrow 0$ and $h_{k} \rightarrow 0$ then the previous equations converge respectively to:

$$
\frac{\partial L}{\partial q}-\frac{d}{d t}\left(\frac{\partial L}{\partial \dot{q}}\right)=0 \quad \text { and } \quad \frac{d}{d t} E_{L}=-\frac{\partial L}{\partial t}
$$

As an alternative way, we consider the discretization of the homogeneous lagrangian:

$$
\mathcal{L}_{d}\left(t_{k}, q_{k}, t_{k+1}, q_{k+1}\right)=\frac{t_{k+1}-t_{k}}{\bar{h}} L\left(\frac{t_{k}+t_{k+1}}{2}, \frac{q_{k}+q_{k+1}}{2}, \frac{q_{k+1}-q_{k}}{t_{k+1}-t_{k}}\right)
$$

where $\bar{h} \in \mathbb{R}_{+}$is the time step. We then extremize

$$
S=\sum_{k=0}^{N-1} \mathcal{L}_{d}\left(t_{k}, q_{k}, t_{k+1}, q_{k+1}\right)=\frac{1}{\bar{h}} \sum_{k=0}^{N-1}\left(t_{k+1}-t_{k}\right) L\left(\frac{t_{k}+t_{k+1}}{2}, \frac{q_{k}+q_{k+1}}{2}, \frac{q_{k+1}-q_{k}}{t_{k+1}-t_{k}}\right)
$$

to derive equations (11) and (2) for the function

$$
L_{d}\left(t_{k}, q_{k}, t_{k+1}, q_{k+1}\right)=L\left(\frac{t_{k}+t_{k+1}}{2}, \frac{q_{k}+q_{k+1}}{2}, \frac{q_{k+1}-q_{k}}{t_{k+1}-t_{k}}\right)
$$

The above formalism is useful since now we can use the results of Chapter 6 of [5] to analyze the local truncation and global error for (11) and (2) as a particular case. 


\subsection{Symplecticity of the algorithm}

Now, we will show that the algorithm (11) is symplectic in a natural way. By similar arguments to those used in [6], it is easy to prove that for solutions of the algorithm (1) without imposing endpoints conditions:

$$
\begin{aligned}
d \mathcal{S}_{\text {solutions }}= & \left(t_{1}-t_{0}\right) D_{2} L_{d}\left(t_{0}, q_{0}, t_{1}, q_{1}\right)+\left(t_{N}-t_{N-1}\right) D_{4} L_{d}\left(t_{N-1}, q_{N-1}, t_{N}, q_{N}\right) \\
& +\left(t_{1}-t_{0}\right) D_{1} L_{d}\left(t_{0}, q_{0}, t_{1}, q_{1}\right)-L_{d}\left(t_{0}, q_{0}, t_{1}, q_{1}\right) d t_{0} \\
& +\left(t_{N}-t_{N-1}\right) D_{3} L_{d}\left(t_{N-1}, q_{N-1}, t_{N}, q_{N}\right)+L_{d}\left(t_{N-1}, q_{N-1}, t_{N}, q_{N}\right) d t_{N} \\
= & \left(t_{1}-t_{0}\right) D_{2} L_{d}\left(t_{0}, q_{0}, t_{1}, q_{1}\right)+\left(t_{N}-t_{N-1}\right) D_{4} L_{d}\left(t_{N-1}, q_{N-1}, t_{N}, q_{N}\right) \\
& +h_{0} D_{1} \bar{L}_{d}\left(t_{0}, q_{0}, h_{0}, q_{1}\right)+E_{d}\left(t_{0}, q_{0}, h_{0}, q_{1}\right) d t_{0} \\
& -E_{d}\left(t_{N-1}, q_{N-1}, h_{N-1}, q_{N}\right) d t_{N} .
\end{aligned}
$$

where $h_{k}=t_{k+1}-t_{k}$. If we put

$$
\begin{aligned}
\text { Energy }_{t_{0}} & =h_{0} \frac{\partial \bar{L}_{d}}{\partial t_{0}}\left(t_{0}, q_{0}, h_{0}, q_{1}\right)+E_{d}\left(t_{0}, q_{0}, h_{0}, q_{1}\right) \\
\text { Energy }_{t_{N}} & =E_{d}\left(t_{N-1}, q_{N-1}, h_{N-1}, v_{N}\right)
\end{aligned}
$$

then

$$
\begin{aligned}
d S_{\mid \text {solutions }}= & h_{0} D_{2} \bar{L}_{d}\left(t_{0}, q_{0}, t_{1}, q_{1}\right)+h_{N-1} D_{4} \bar{L}_{d}\left(t_{N-1}, q_{N-1}, h_{N-1}, q_{N}\right) \\
& + \text { Energy }_{t_{0}} d t_{0}-\text { Energy }_{t_{N}} d t_{N} .
\end{aligned}
$$

Observe the similarity of this expression with the classical transversality conditions for variable initial and terminal points when we are studying extremals of functionals determined by time-dependent lagrangian systems.

Now, fix $N=2$ and consider the 1-forms $\Theta_{L_{d}}^{-}$and $\Theta_{L_{d}}^{+}$following a similar definition than in Marsden et al 14]:

$$
\begin{aligned}
\Theta_{L_{d}}^{-}\left(t_{0}, q_{0}, t_{1}, q_{1}\right)= & \left(t_{1}-t_{0}\right) D_{2} L_{d}\left(t_{0}, q_{0}, t_{1}, q_{1}\right) \\
& +\left(t_{1}-t_{0}\right) D_{1} L_{d}\left(t_{0}, q_{0}, t_{1}, q_{1}\right)-L_{d}\left(t_{0}, q_{0}, t_{1}, q_{1}\right) d t_{0} \\
\Theta_{L_{d}}^{+}\left(t_{0}, q_{0}, t_{1}, q_{1}\right)= & \left(t_{1}-t_{0}\right) D_{4} L_{d}\left(t_{0}, q_{0}, t_{1}, q_{1}\right) \\
& +\left(t_{1}-t_{0}\right) D_{3} L_{d}\left(t_{0}, q_{0}, t_{1}, q_{1}\right)+L_{d}\left(t_{0}, q_{0}, t_{1}, q_{1}\right) d t_{1} .
\end{aligned}
$$

In view of the equations defining the algorithm, we deduce that

$$
d \mathcal{S}_{\mid \text {solutions }}=\Theta_{L_{d}}^{-}+\Phi^{*} \Theta_{L_{d}}^{+}
$$

Since $d d \mathcal{S}=0$, we have $d \Theta_{L_{d}}^{-}=-\Phi^{*} d \Theta_{L_{d}}^{+}$, but observe that $\Theta_{L_{d}}^{-}+\Theta_{L_{d}}^{+}=d\left(\left(t_{1}-t_{0}\right) L_{d}\right)$, that is, $d \Theta_{L_{d}}^{-}+d \Theta_{L_{d}}^{+}=0$ and, consequently, $\Phi^{*} \Omega_{d}=\Omega_{d}$ where $\Omega_{d}=d \Theta_{L_{d}}^{-}=-d \Theta_{L_{d}}^{+}$. 
Notice that $\Omega_{d}=-d \Theta_{L_{d}}^{+}$, where $\Theta_{L_{d}}^{+}$is equal to

$$
h_{0} D_{4} L_{d}\left(t_{0}, q_{0}, t_{1}, q_{1}\right)-E_{d}\left(t_{0}, q_{0}, h_{0}, q_{1}\right) d t_{1}
$$

with $t_{0}+h_{0}=t_{1}$.

The 2-form $\Omega_{d}$ is symplectic if and only if the matrix $\left(\bar{D}_{12} L_{d}\right)$ is regular. Observe also the similarity of the constructions of $\Theta_{L_{d}}^{+}$and $\Omega_{d}$ with the Poincaré-Cartan 1-form and 2-form, respectively, for time-dependent lagrangian systems (see [12, 18])

\subsection{Momentum conservation}

The conservation of momentum for an algorithm invariant under a symmetry group follows in the same way as usual [20] considering here the invariance of the function $\left(t_{k+1}-t_{k}\right) L_{d}\left(t_{k}, q_{k}, t_{k+1}, q_{k+1}\right)$.

In particular, we suppose that $\left(t_{k+1}-t_{k}\right) L_{d}\left(t_{k}, q_{k}, t_{k+1}, q_{k+1}\right)$ is $G$-invariant under the diagonal action of a Lie group $G$ on $\mathbb{R} \times Q$. Denote by $\Phi_{g}: \mathbb{R} \times Q \longrightarrow \mathbb{R} \times Q$ the group action, $g \in G$. For any element $\xi$ in the Lie algebra $\mathfrak{g}$ of $G$, we denote by $\xi_{\mathbb{R} \times Q}$ the fundamental vector field which generates the flow $\Phi_{\exp (s \xi)}: \mathbb{R} \times Q \rightarrow \mathbb{R} \times Q$. Moreover $\xi_{\mathbb{R} \times Q}(t, q)=\xi_{\mathbb{R}}(t, q)+\xi_{Q}(t, q)$ where $\xi_{\mathbb{R}}(t, q) \in T_{t} \mathbb{R}$ and $\xi_{Q}(t, q) \in T_{q} Q$.

Therefore, applying the results for discrete algorithms for conservative mechanical systems (see for instance 20]) we obtain that the momentum map $J_{d}:(\mathbb{R} \times Q)^{2} \rightarrow \mathfrak{g}^{*}$ defined by

$$
\left\langle J_{d}\left(t_{k}, q_{k}, t_{k+1}, q_{k+1}\right), \xi\right\rangle=\left\langle\bar{D}_{2}\left[\left(t_{k+1}-t_{k}\right) L_{d}\left(t_{k}, q_{k}, t_{k+1}, q_{k+1}\right)\right], \xi_{\mathbb{R} \times Q}\left(t_{k+1}, q_{k+1}\right)\right\rangle
$$

is preserved by the discrete flow $\Phi:(\mathbb{R} \times Q)^{2} \longrightarrow(\mathbb{R} \times Q)^{2}$.

\section{Symplectic-Energy-Momentum preserving variational integrators}

In Kane, Marsden and Ortiz [6] it is constructed a variational integrator for conservative mechanical systems with time-step adaptation. Their algorithm is symplectic, energy and momentum conserving. We will show that the Kane, Marsden and Ortiz's algorithm is a particular case of ours when we impose that the discrete lagrangian only depends on the time step $\left(h_{k}=t_{k+1}-t_{k}\right)$.

Assume that $L_{d}\left(t_{0}, q_{0}, t_{1}, q_{1}\right)=\bar{L}_{d}\left(q_{0}, q_{1}, t_{1}-t_{0}\right)=\bar{L}_{d}\left(q_{0}, q_{1}, h_{0}\right)$ and, therefore, $D_{1} \bar{L}_{d}=D_{2} L_{d}, D_{2} \bar{L}_{d}=D_{4} L_{d}$, and

$$
\frac{\partial \bar{L}_{d}}{\partial h}\left(q_{0}, q_{1}, t_{1}-t_{0}\right)=-\frac{\partial L_{d}}{\partial t_{0}}\left(t_{0}, q_{0}, t_{1}, q_{1}\right)=\frac{\partial L_{d}}{\partial t_{1}}\left(t_{0}, q_{0}, t_{1}, q_{1}\right)
$$

Then, equations (11) and (2) can be rewritten as

$$
\begin{aligned}
h_{k} D_{1} \bar{L}_{d}\left(q_{k}, q_{k+1}, h_{k}\right)+h_{k-1} D_{2} \bar{L}_{d}\left(q_{k-1}, q_{k}, h_{k-1}\right) & =0 \\
E_{d}\left(q_{k}, q_{k+1}, h_{k}\right)-E_{d}\left(q_{k-1}, q_{k}, h_{k-1}\right) & =0
\end{aligned}
$$

which are exactly the equations proposed in [6].

Observe that in this case the discrete lagrangian $L_{d}$ is invariant by time translations, and the associated conservation law is precisely the energy. 


\section{Concluding remarks}

This natural variational interpretation of the results of [6] was independently obtained by Chen, Gou, $\mathrm{Wu}$ (see [2]). They use Lee's approach and also derive in a variational way the symplectic-energy-momentum integrator for conservative lagrangian systems.

The authors wish to thank an anonymous referee for valuable and useful comments and for pointing out to us reference [2].

\section{Acknowledgments}

This work has been partially supported by grants DGICYT (Spain), PB97-1257 and PGC2000-2191-E.

\section{REFERENCES}

[1] J. Cortés and S. Martínez: Nonlinearity 14, 1365-1392 (2001).

[2] J.B. Chen, H-Y. Guo, K. Wu: arxiv:hepo-th/0109178, 23 sep 2001.

[3] F. Cantrijn, J.F. Cariñena, M. Crampin, A. Ibort: J. Geom. Phys. 3 (3), 353-400 (1986)

[4] Z. Ge and J. Marsden: Phys. Lett. A 133, 134-139 (1988).

[5] J. Gregory, C. Lin: Constrained Optimization in the Calculus of Variations and Optimal Control Theory, Chapman\& Hall, London 1992.

[6] C. Kane, J. Marsden, M. Ortiz, M. West: J. Math. Phys., 40, 3353-3371 (1999).

[7] H. Kataoka, H. Hashimoto: J. Math. Econ. 36 (8), 271-280 (1995).

[8] T.D. Lee: Phys. Lett., 122B, 217-220 (1983).

[9] T.D. Lee: J. Statis. Phys., 46, 843-860 (1987).

[10] T.D. Lee: Discrete mechanics, Lectures given at the International School of Subnuclear Physics, Erice, August 1983.

[11] M. de León, J.C. Marrero and D. Martín de Diego: J. Geom. Phys. 35, 126-144 (2000).

[12] M. de León and P.R. Rodrigues: Methods of Differential Geometry in Analytical Mechanics. North-Holland Math. Ser. 152, Amsterdam 1989.

[13] M.J.P. Magill: On a General Economic Theory of Motion, Springer-Verlag, Berlin 1970.

[14] J.E. Marsden, G.W. Patrick and S. Shkoller: Comm. Math. Phys., 199, 351-395 (1988).

[15] F.P. Ramsey: Economic J. 38 543-559 (1928).

[16] J.M. Sanz-Serna and M. Calvo: Numerical Hamiltonian problems, Chapman and Hall, London 1994.

[17] R. Sato and R.V. Ramachandran: Conservation Laws and Symmetry: Applications to Economics and Finance, Kluwer, Boston 1990.

[18] D. J. Saunders: The Geometry of Jet Bundles, London Math. Soc. Lect. Notes Series 142, Cambridge University Press, 1989.

[19] A.P. Veselov: Funct. An. and Appl. 25, 112-123 (1991).

[20] J.M. Wendlandt and J.E. Marsden: Physica D 106 223-246 (1997). 